Comienzan a ser frecuentes los estudios llevados a cabo en nues tro país sobre el miedo al delito. Los trabajos empíicos realizados en otros países tratan de conocer algo más acerca este fenómeno, cada vez más consistente, a tavés del análisis de su etiología, incidencia, caracteństicas, distribución espacial y consecuencias. $\mathrm{\theta}$ trabajo que aquí se presenta incide precisamente a tavés de un sofisticado sistema de información geográfica, en el estudio, de la relación entre el miedo al delito y los escenarios urbanos. A pesar de que tendemos a asociar el miedo al delito con el deterioro ambiental, lo cierto es que en este trabajo se muestra la correlación existente entre entomos ambientales no deteriorados y miedo al delito, cumpliéndose así la denominada "paradoja del miedo al delito". Sus autores son profesores del Instituto Vasco de Criminología (Universidad del País Vasco) Se ha realizado con financiación del gobiemo vasco, a través de la concesión de una beca del programa de formación de investigadores del departamento de Educación, Universidades e Investigación. Ha sido galardonado con el Premio SEIC a la promoción de investigadores noveles en Criminología. La versión completa de este trabajo se publicará próximamente en la Revista Española de Investigación Criminológica (REC).

Palabras clave: Miedo al delito, inseguridad ciudadana, escenanos urbanos, sistemas de información geográfica.

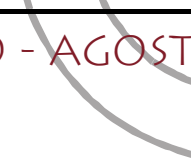

\title{
SISTEMAS DE INFORMACIÓN GEOGRÁFICA EN EL ESTUDIO DEL MIEDO AL DELITO: EL CASO DE DONOSTIA-SAN SEBASTIÁN
}

\section{Laura Vozmediano Sanz César San Juan Guillén}

\section{Miedo al delito y escenarios} urbanos

El miedo al delito ha sido ampliamente estudiado en Criminología desde los años 70, aunque abordado previamente desde otras disciplinas tales como la sociología, la geografía, el urbanismo, la arquitectura y la psicología social y ambiental (Fernández y Corraliza, 1998), (Carro, Valera y Vidal, 2005). Se trata de un área de trabajo que es inevitablemente muldisciplinar, toda vez que tanto los factores que originan el miedo al delito, como las consecuencias del propio miedo, implican a todas las áreas mencionadas. Su explicación, lejos de resultar sencilla, parece responder a una multiplicidad de factores que en la actualidad tratamos de integrar para retratar de modo coherente la génesis y el mantenimiento de este miedo. Sus consecuencias, asimismo, son diversas: ansiedad personal, debilitamiento de los vínculos sociales o detrimento de la calidad de vida urbana, son algunas de ellas.

Diversos investigadores de nuestro país han aportado elementos de discusión a este tema desde la Psicología social y ambiental, realizando interesantes contribuciones a nivel teórico y empírico. En los últimos tiempos, el debate se centra en tres grupos de variables, con las que se pretende configurar las coordenadas de la génesis y el mantenimiento del miedo al delito: variables personales, sociales y ambientales.

Las variables personales serían el sexo, edad, capacidades de afrontamiento y control; en definitiva, facto- res de vulnerabilidad relativa frente al delito. Aunque no podemos olvidar que la prueba empírica disponible de algunas variables es, en ocasiones, contradictoria.

Las variables psicosociales se relacionan con la dinámica y cohesión vecinales, que podrían ser protectoras frente el miedo al delito al incrementar el control social. Se han utilizado variables como la satisfacción residencial, apego al barrio, y sentido de comunidad, aunque en algunos trabajos estas variables mostraban un escaso poder predictivo frente a posicionamientos más razonados, tales como la confianza en la acción policial y la justicia (San Juan, Vergara y Germán, 2005).

Finalmente, podemos distinguir dos niveles o clases de variables ambientales relevantes en relación al miedo al delito, y al delito mismo. Por un lado, las que hacen referencia a la estructura del espacio urbano: Las características estructurales de un lugar podrían inhibir las relaciones sociales, hacerlo más propenso a la ocurrencia de delitos, y elicitar más fácilmente miedo al delito; se trataría por tanto de un "espacio crimípeto" (San Juan, 2000). Por otro lado, las relacionadas con la degradación de los espacios, en la línea de la perspectiva de las "incivilidades" (Lewis y Maxfield, 1980) y de la teoría de las "ventanas rotas" (Wilson y Kelling, 1982).

Los tres grupos de variables mencionadas (personales, sociales y ambientales) configuran la perspectiva psico-socio-ambiental desde la que 


\section{METO DO LO G ÍA}

504 sujetos residentes en San Sebastián, distribuidos en tres grupos con igual número de integrantes, y residiendo cada grupo en uno de los esc enarios que componen el estudio, fueron entrevistados en sus domicilios. Respondieron a un cuestionario que incluía las variables siguientes: miedo al delito, victimación, satisfacción con sus vecinos, satisfacción con el entomo físico, juicio sobre el nivel de inseguridad del bamio en comparación con otros de la ciudad, satisfacción con la actuación de los jueces frente a la inseguridad ciudadana, y satisfacción con la política de seguridad del ayuntamiento.

Los escenarios fueron 3 bamios de la ciudad, concretamente los denominados Centro, Amara y Alza, que fueron seleccionados como prototípicos de tres diferentes niveles socio-económic os, alto, medio y bajo res pectivamente. El género estuvo equilibrado al 50\%, estableciéndose la media de edad en los 48 años.

Adicionalmente, y con intención de comparar el miedo al delito con la distribución de la delincuencia objetiva en la ciudad, se tomaron datos de un estudio sobre la criminalidad registrada judicialmente en Guipúzcoa, del cual se emplearon las sentencias referidas a delitos ocumidos en la ciudad de Donostia-San Sebastián, 311 en total.

En cuanto al marco espacial del trabajo, la ciudad de Donostia-San Sebastián, se empleó un mapa digital que el Gobiemo Vasco pone a disposición de los ciudadanos en su página web. Correos proporcionó un mapa en papel con los límites de los códigos postales, a partir del cual se construyó un mapa digital con esta zonificación. La combinación de estos mapas con los datos de las encuestas y sentencias, en el programa ArcGIS 9.0, permitió elaborar los mapas temáticos que fueron analizados en el estudio.

abordamos el análisis del miedo al delito. Con todo en este estudio concreto, nos centramos en la relevancia de las variables ambientales.

La hipótesis de partida fue que las características de los espacios influirían en el miedo a ser víctima de un delito. Por tanto, tomando los distintos escenarios de la ciudad como elemento de análisis, hipotetizamos que distintos escenarios elicitarían distintos niveles de miedo. Es decir, la distribución del miedo al delito en los diversos escenarios de la ciudad no sería homogénea. El primer objetivo, por tanto, es determinar si esto se cumple. Pero además, para poder afirmar que la perspectiva psico-socioambiental es relevante, sería necesario establecer que dicha distribución no homogénea no se debe a una hipótesis rival: la distribución del delito mismo, que tampoco será homogénea. Para comprobar este aspecto, un segundo objetivo es analizar la distribución del delito registrado judicialmente, y la distribución de la victimación (ambos, indicadores de la distribución objetiva del delito).

Para comprobar si estas dos cuestiones se cumplen en una urbe concreta -la ciudad de Donostia-San Sebastián fue la elegida-, el equipo consideró que un software SIG era la herramienta adecuada, ya que nos permitiría construir los mapas del delito y del miedo al delito en los escenarios estudiados.

SIG para el análisis espacial de los fenómenos sociales

La tecnología SIG (Sistemas de Información Geográfica) pretende ser útil para el estudio y búsqueda de soluciones de problemas del mundo real, trabajando sobre un modelo cartográfico de dicha realidad. Integra las ventajas de las bases de datos y de los mapas tradicionales, en un sistema informatizado que nos permite trabajar no sólo con información, sino con información geo-referenciada. Es, por tanto, aplicable a multitud de campos de trabajo y estudio; en nuestro caso, encontramos particularmente interesante su aplicación para el estudio de la dimensión geográfica de los fenómenos sociales.

Concretamente, en el estudio del fenómeno delictivo su uso se halla muy extendido en los ámbitos académico, administrativo y policial. Un sistema de este tipo nos permitiría construir una base de datos que contenga, por ejemplo, el callejero de una ciudad, datos sociodemográficos de sus habitantes con una referencia espacial (la dirección del censo), datos sobre servicios en cada zona (colegios, bibliotecas, comisarías...) y datos de delitos denunciados con su referencia espacial (lugar de comisión del supuesto delito). Bases de datos de este tipo se emplean para la elaboración de "mapas del delito", que nos muestran cómo se distribuyen distintos tipos de delitos por la ciudad, y permiten relacionar ese patrón con otras variables. Asimismo, se utilizan para gestionar la respuesta policial en las llamadas de denuncia, o para planificar las necesidades de agentes en las distintas zonas de las ciudades. Además, es cada vez más usual el empleo de estos sistemas para comunicar a la sociedad la situación en relación a los delitos, y para recibir información de los ciudadanos a través de Internet. En definitiva, el SIG favorece la comprensión y prevención del delito y ha demostrado su utilidad en muchos países (Weisburd y McEwen, 1997).
En nuestro país su empleo es creciente. Stangeland y Garrido de los Santos (2004), por ejemplo, lo emplearon en el análisis de la delincuencia en Málaga, con satisfactorios resultados. Sin embargo, su uso no tiene porqué limitarse al estudio de la delincuencia objetiva, y los mismos autores sugieren la aplicabilidad del sistema para el estudio del miedo al delito. Del mismo modo que se construyen los mapas de delito, es posible construir mapas del miedo al delito, y comparar ambos, posibilitando así alcanzar los objetivos que habíamos marcado para este trabajo.

\section{Resultados}

El resultado del proceso descrito fueron una serie de mapas. El primero de ellos se denominó "mapa de densidad de delito". En él se representó, para cada una de las parcelas del mapa (correspondientes a los códigos postales) el número total de delitos según los datos judiciales, dividido por la extensión de la parcela. Este índice de densidad de delito permitió establecer comparaciones entre parcelas a pesar de la disparidad de área de cada una de ellas. Ya que este mapa abarca toda la ciudad objeto de estudio, fue el marco sobre el que se muestran los mapas de victimación (figura 1) y de miedo al delito (figura 2). Estos dos últimos mapas contienen los tres escenarios del estudio en los que se encuestó a los ciudadanos sobre victimación y miedo al delito.

En la figura 1 podemos apreciar, respecto a la distribución del delito registrado judicialmente, que la mayor densidad de delito se agrupa en las zonas más céntricas de la ciudad. La victimación declarada por los sujetos del estudio se distribuye de modo coherente con el índice anterior: Los 


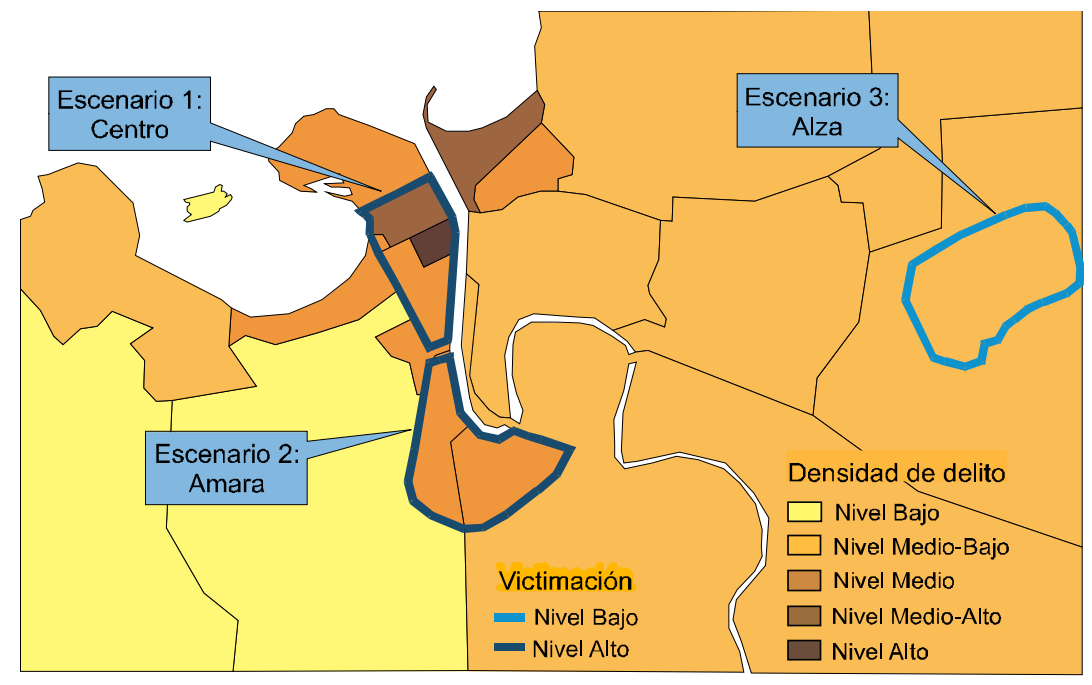

Figura 1. Distribución de la densidad de delito y de la victimación

escenarios 1 y 2, emplazados en áreas con mayor densidad de delito, acogen a sujetos que tienen un índice de victimación superior a los residentes en el escenario 3 , siendo esta diferencia estadísticamente significativa.

En la figura 2 se observa que el miedo al delito, por el contrario, no es más intenso en los escenarios 1 y 2 . Ocurre lo inverso, los residentes en el escenario 3 tienen un miedo al delito significativamente superior que los que habitan en el 1 y 2. Esta distribución no se corresponde, por tanto, con la del delito, bien sea éste recogido de las sentencias judiciales, bien de la encuestas de victimación. Téngase en cuenta que son los mismos sujetos que reportaron, como media, menor victimación, los que declaran mayor miedo al delito, puesto que ambas variables se midieron en la misma muestra.

Tomando en cuenta que el miedo al delito superior en el escenario 3 no se relacionaba con la densidad de delito registrado judicialmente ni con la victimación, se exploró la distribución en los tres barrios de las variables psico-socio-ambientales recogidas en el cuestionario: satisfacción con el espacio físico, satisfacción con los vecinos, inseguridad relativa, satisfacción con la actuación de los jueces frente a la inseguridad ciudadana y satisfacción con la política de seguridad del ayuntamiento. Los residentes en el escenario 3 mostraron: menor satisfacción con el espacio físico que los otros dos escenarios; menor satisfacción con los vecinos que el escenario 1, mayor percepción de su barrio como inseguro, en comparación con otros, que los otros dos escenarios; y menor satisfacción con las actuaciones judiciales y con la política del ayuntamiento que los otros dos escenarios. Todas estas diferencias fueron estadísticamente significativas con al menos $\mathrm{p}<0.05$.

\section{Discusión}

El análisis de los mapas obtenidos revela una falta de corresponden-

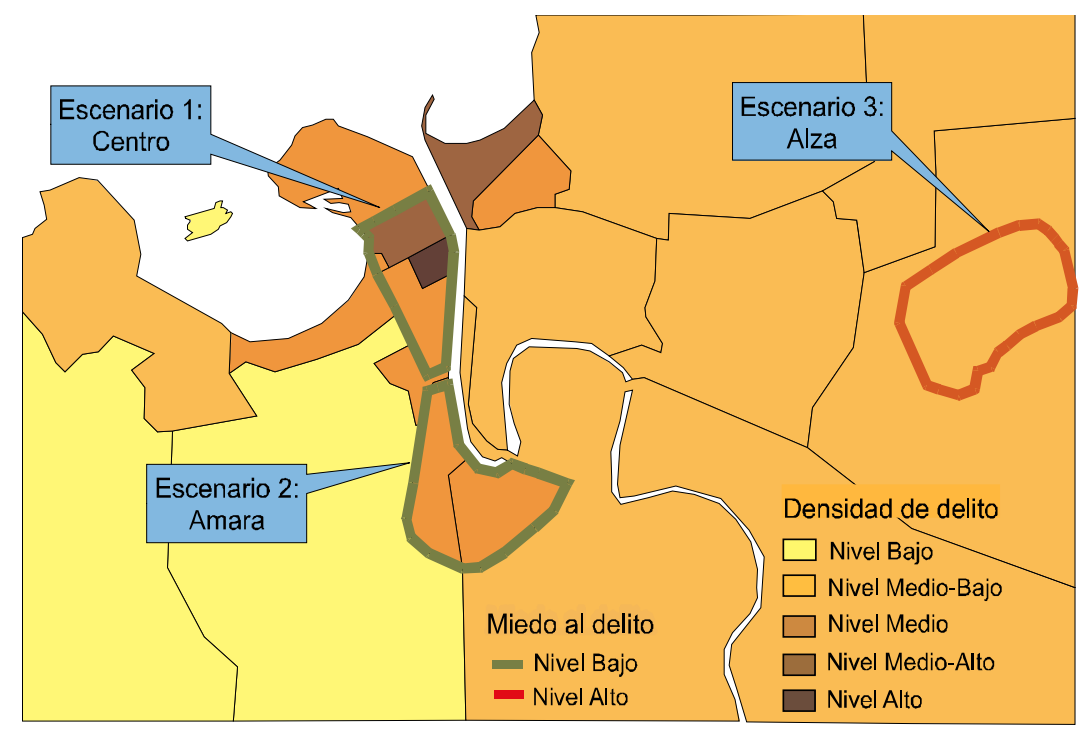

Figura 2. Distribución de la densidad de delito y del miedo al delito

cia en la localización geográfica entre los escenarios en los que hay más miedo al delito, y aquellos que efectivamente soportan un índice de delitos mayor. Estos resultados se pueden discutir a la luz de diversas aportaciones teóricas, que nos ayudarían a clarificar su relevancia y alcance.

En primer lugar, los resultados se puede interpretar en términos de la denominada paradoja del miedo al delito (Fattah, 1993). La falta de correspondencia entre los elementos objetivos de la realidad, y los subjetivos del miedo al delito, constituye un fenómeno frecuente pero, en todo caso, no absolutamente generalizable. Los resultados son en ocasiones contradictorios, poniendo posiblemente en evidencia la importancia de la validez ecológica de este tipo de investigaciones.

En nuestro estudio existe, en todo caso, una correlación negativa entre delincuencia objetiva y miedo al delito subjetivo que merece ser tenida en cuenta a la hora de explicar el fenómeno de la percepción de inseguridad desde una perspectiva psico-socioambiental. Dicha perspectiva podría incluso explicar la disparidad de resultados mencionada. La influencia de las características de los espacios dibujaría en cada contexto urbano estudiado un patrón de miedo al delito propio, coincidente, en unos casos con el delito objetivo, pero no en otros. Otro elemento que puede influir en la disparidad de resultados al respecto es el desplazamiento del fenómeno delictivo en la ciudad. Desde una perspectiva espacio-temporal, una zona que ha soportado altos índices de delito en el pasado podría mantener un mayor nivel de miedo al delito aun cuando el delito haya "migrado" a otra zona de la ciudad como consecuencia, por ejemplo, de la intensificación puntual de la acción policial. La percepción de ese espacio como peligroso podría perdurar más allá de la persistencia de elementos objetivos, una vez que se ha incorporado a la dinámica de la vida cotidiana en un vecindario concreto.

Por otro lado, las variables psico-socio-ambientales incluidas en el estudio sugieren que la percepción del espacio físico por los vecinos, así como la creencias compartidas sobre la efectividad de la justicia y el papel del ayuntamiento en garantizar la seguridad, podrían estar jugando un papel en la génesis y mantenimiento 
del miedo al delito. Claro que los factores estructurales del espacio son difícilmente aislables en la investigación. Es evidente que las configuraciones urbanísticas con una visión más funcional, o que no responden a patrones de tipo estético, suelen ser el escenario de convivencia de las clases más desfavorecidas, de modo que las diferencias obtenidas en la percepción de miedo podrían estar condicionadas por el estatus socioeconómico antes que por elementos de configuración del espacio.

Tratándose de un estudio exploratorio, que busca abrir una línea de trabajo, no cabe hacer afirmaciones tajantes, pero nuestros resultados sugieren la pertinencia de seguir investigando la influencia de estas variables en la percepción de inseguridad, en general, y en la valoración de la probabilidad de ser víctima de un delito, en particular.

Entre las tareas a abordar en el futuro, por tanto, nos encontramos con la necesidad de establecer la relevancia de las distintas variables psicosocio-ambientales, introduciendo, además, el elemento espacio-temporal en el análisis. Asimismo sería necesario comparar los patrones de miedo al delito de distintas ciudades y, en una misma ciudad, de distintos delitos, estableciendo qué elementos son propios y característicos de ciertas urbes, o de ciertos delitos, y cuáles son los elementos comunes. La importancia de estos últimos radica en su potencia- lidad para intervenir en la reducción y prevención del miedo, con el objetivo último de incrementar la calidad de vida urbana.

Respecto al empleo de la aplicación SIG en el estudio del miedo al delito, el trabajo llevado a cabo nos permitió realizar una pequeña evaluación de las potencialidades y dificultades que conlleva su uso. Podemos afirmar que la experiencia ha resultado positiva, toda vez que nos ha posibilitado construir el mapa del delito y del miedo al delito en tres escenarios diferentes de un mismo contexto urbano. La explotación de datos con esta aplicación tiene una ventaja adicional muy pertinente desde el punto de vista de la comunicación, ya que se pueden presentar los resultados mediante sucesivas representaciones cartográficas temáticas. En este sentido, puede ser particularmente importante su aplicabilidad para el asesoramiento en la toma de decisiones sobre políticas de seguridad. Un tercer elemento positivo es la amplitud de posibilidades que ofrece el empleo de esta herramienta para la exploración de nuevas ideas y contrastación de hipótesis. Cualquier otra información referenciada geográficamente, que pueda tener relevancia es susceptible de ser incluida en el estudio. Así, por ejemplo, se podrían incluir la localización de pasos subterráneos y de parques, localización de comisarías, número de efectivos policiales asignados a cada área, diferenciadas tipologías delicti- vas, etc.

Cabe decir que la principal limitación para el empleo de esta tecnología consiste en la necesidad de disponer de una cartografía en formato digital de referencia del escenario geográfico objeto de estudio que sea compatible con el programa. Por otra parte, sería necesario hacer algunos ajustes en los datos de frecuencia de delitos registrados que se reflejan en la representación cartográfica, ya que son simples indicadores de prevalencia que no contemplan la ratio por densidad de población o grado de urbanización.

Al margen de estas limitaciones, podemos afirmar que este estudio exploratorio consigue poner de manifiesto la relevancia de los escenarios y sus características sociales y ambientales en el miedo al delito urbano, y lo hace utilizando una herramienta que produce representaciones amigables, que pueden ser fácilmente comprendidas y empleadas para la toma de decisiones. Además, las representaciones espaciales de los datos favorecen nuevos caminos para la reflexión y el descubrimiento de relaciones entre las variables. Pensamos, por tanto, que el empleo de SIG en el futuro permitirá abordar una serie de cuestiones que permanecen sin resolver, en relación al miedo al delito. Y que será de ayuda en las tareas encaminadas a su contención.

\section{Referencias bibliográficas}

Carro, D., Valera, S. y Vidal, T. (2005). Inseguridad percibida en el espacio público: variables personales, sociales y ambientales en un estudio de un barrio de Barcelona. En R. García Mira, A. Fernández González, M. D. Losada Otero y M. Golugoff Scheps (Comps.). Psicología Ambiental, Comunitaria y de la Educación. Madrid: Biblioteca Nueva.

Fattah, E. A. (1993). Research on Fear of Crime: Some Common Conceptual and Measurement Problems. En W. Bilsky, Ch. Pfeiffer y P. Wetzels (Eds.), Fear of Crime and Criminal Victimization. Stuttgart: Enke.

Fernández Ramírez, B. y Corraliza, J. A. (1998), Generalidad y especificidad en la explicación del miedo al delito. Apuntes de Psicología, 16 (1-2): 173-186.

Lewis, D. y Maxfield, M. (1980). Fear in the Neighbourhoods: An Investigation of the Impact of Crime. Journal of Research in Crime and Delinquency, 17: 160-89.

San Juan, C. (2000): Theories of Design and Designs of Theory in the Environmental Interventions. Bulletin of People Environment Studies, 15: 15-16.

San Juan, C., Vergara, A. y Germán, I. (2005). Propiedades psicométricas de un cuestionario para la evaluación de la calidad de vida urbana y el miedo al delito. Revista Española de Investigación Criminológica, 3: 1-13.

http://www.criminologia.net/Revista.htm

Stangeland, P. y Garrido de los santos, M. J. (2004). El mapa del crimen. Valencia: Tirant lo blanch.

Weisburd, D. y McEwen, T. (1997). Crime Mapping and Crime Prevention. Monsey, New York: Criminal Justice Press.

Wilson, J. Q. y Kelling, G. L. (1982). The Police and Neighborhood Safety: Broken Windows. The atlantic Monthly, 249: 29-38. 